University of Wollongong

Research Online

Faculty of Engineering and Information

Faculty of Engineering and Information

Sciences - Papers: Part A

Sciences

$1-1-2014$

\title{
Closed-orbit dependence on the field direction in the anisotropic diamagnetic Kepler problem
}

\author{
A Bruno-Alfonso \\ University of Wollongong \\ Colin Bleasdale \\ University of Wollongong, cb123@uowmail.edu.au \\ Gustavo Alvarez \\ University of Wollongong, gustavo@uow.edu.au
}

R A. Lewis

University of Wollongong, roger@uow.edu.au

Follow this and additional works at: https://ro.uow.edu.au/eispapers

Part of the Engineering Commons, and the Science and Technology Studies Commons

Research Online is the open access institutional repository for the University of Wollongong. For further information contact the UOW Library: research-pubs@uow.edu.au 


\title{
Closed-orbit dependence on the field direction in the anisotropic diamagnetic Kepler problem
}

\author{
Abstract \\ The classical orbits of an electron with anisotropic mass interacting with a Coulomb center in the \\ presence of a magnetic field are investigated. It is shown that the shape and duration of the closed orbits \\ depend on the magnetic-field direction. Reasonable agreement with available experimental results for \\ donor-doped silicon samples in a magnetic field is found, when the energy value of the conduction \\ electron is appropriately chosen.

\section{Keywords} \\ kepler, diamagnetic, anisotropic, direction, field, problem, dependence, closed, orbit \\ Disciplines \\ Engineering | Science and Technology Studies

\section{Publication Details} \\ Bruno-Alfonso, A., Bleasdale, C., de Souza, G. V. B. \& Lewis, R. A. (2014). Closed-orbit dependence on the \\ field direction in the anisotropic diamagnetic Kepler problem. Physical Review A: Atomic, Molecular and \\ Optical Physics, 89 043425-1-043425-6.
}




\title{
Closed-orbit dependence on the field direction in the anisotropic diamagnetic Kepler problem
}

\author{
A. Bruno-Alfonso, ${ }^{1,2,{ }^{*}}$ C. Bleasdale, ${ }^{1}$ G. V. B. de Souza, ${ }^{1,3}$ and R. A. Lewis ${ }^{1}$ \\ ${ }^{1}$ Institute for Superconducting and Electronic Materials and School of Physics, University of Wollongong, New South Wales, 2522, Australia \\ ${ }^{2}$ Departamento de Matemática, Faculdade de Ciências, Unesp - Universidade Estadual Paulista, Bauru, SP, 17033-360, Brazil \\ ${ }^{3}$ POSMAT, Unesp - Universidade Estadual Paulista, Bauru, SP, 17033-360, Brazil
}

(Received 19 December 2013; revised manuscript received 27 March 2014; published 23 April 2014)

\begin{abstract}
The classical orbits of an electron with anisotropic mass interacting with a Coulomb center in the presence of a magnetic field are investigated. It is shown that the shape and duration of the closed orbits depend on the magnetic-field direction. Reasonable agreement with available experimental results for donor-doped silicon samples in a magnetic field is found, when the energy value of the conduction electron is appropriately chosen.
\end{abstract}

DOI: 10.1103/PhysRevA.89.043425

PACS number(s): $32.80 . \mathrm{Rm}, 05.45 . \mathrm{Mt}, 71.55 . \mathrm{Cn}$

\section{INTRODUCTION}

The main effects of external electric and magnetic fields on the electronic spectrum of atoms are broadly known as the Stark and Zeeman effects, respectively. In the Stark effect, the energy levels and their lifetimes depend on the electric-field strength. In the Zeeman effect, the atomic degenerate levels split and essentially show a paramagnetic behavior. For the ground and a few tens of excited states, such effects have been calculated with some accuracy since the early developments of quantum mechanics. However, near the ionization threshold, a strong mixing of the unperturbed states occurs and the diamagnetic Zeeman effect may be detected.

In an experimental investigation of the electronic transitions from the low-lying states to the excited states near the continuum, Garton and Tomkins [1] found that the optical absorption of barium atoms in the presence of a magnetic field displays unexpected oscillations as a function of the radiation frequency. The period of the oscillations was found to be two-thirds of the cyclotron period. The subsequent theoretical approaches to this diamagnetic Kepler problem is an essential part of quantum chaology [2-4].

The same kind of oscillation was detected in the optical spectrum of hydrogen by Main et al. [5], who also reported a successful explanation of the peaks in the Fourier transform, namely, they were associated with the closed orbits of the electron. The high density of levels near and above the ionization threshold and the large radius or unboundedness of such quantum states justify the use of a classical approach. Moreover, since the initial states of the relevant electronic transitions are quite localized, the important closed orbits are those departing from and returning to the nucleus. Later, Holle et al. [6] studied the evolution of the spectrum as a function of excitation energy and magnetic-field strength, while Du and Delos [7] presented a detailed investigation of a family of the most important 65 orbits. Wintgen and Friedrich [8] also addressed the problem, giving a classification of the orbits and displaying the correspondence between the classical and quantum results.

Because of the similarity between the atomic orbitals and the envelope functions of donor states in isotropic semiconductors, one may expect the optical spectrum of $n$-doped

\footnotetext{
*alexys@fc.unesp.br
}

semiconductors to also present Garton-Tomkins oscillations. To the best of our knowledge, such oscillations have not been identified in the spectrum of isotropic semiconductors such as GaAs, but very interesting results have recently become available for the case of silicon. Chen et al. [9] have reported photothermal ionization spectroscopy measurements of $n$-doped silicon samples in a magnetic field. When the electronic states of the donor impurities are described within the effective-mass theory, the impurity center plays the role of the atomic nucleus. But in silicon the effective mass is not isotropic and this introduces the so-called anisotropic diamagnetic Kepler problem (ADKP). To deal with this problem in a simple way, those authors proposed an average isotropic model for the pertinent valley of the conduction band, with the electron mass given by the cyclotron mass for each valley of the conduction band. As a result, an identification of the relevant closed orbits of the electron was given.

In the present work we revisit the ADKP for donors in silicon, aiming for a more detailed description of the effects of anisotropy on the relevant electron orbits. In particular, we investigate the dependence of the duration and shapes of the closed orbits on the magnetic-field direction and electron energy. Then we make a new comparison with the available experimental results [9], and discuss whether the experimental conditions are suitable for the identification of the classical orbits. The main text is organized as follows: the basic equations are given in Sec. II, the numerical results are displayed and analyzed in Sec. III, and the main findings are summarized in Sec. IV.

\section{THEORY}

In this section the main equations leading to the closed orbits are presented. Section II A is devoted to solving the problem in the absence of the Coulomb interaction. This leads to the well-known expression for the cyclotron mass and establishes a natural time unit. Additionally, the shapes of the cyclotron orbits are described. In Sec. II B, the Coulomb interaction is included and dimensionless equations of motion are set up. In Sec. II C an appropriate energy scale is introduced and its relation to the quantum mechanical approach is discussed, whereas Sec. II D presents equations for the specific case of a valley in the conduction band of silicon. Finally, the treatment of the Coulomb singularity is given in Sec. II E. 


\section{A. Cyclotron frequency}

First we deal with the motion of an electron with anisotropic mass given by a diagonal matrix $1 / m$ subject to a uniform magnetic field $\boldsymbol{B}$. Since $(1 / m)_{j, j^{\prime}}=m_{j}^{-1} \delta_{j, j^{\prime}}$, the components of the acceleration are given by

$$
m_{j} \ddot{x}_{j}=-e(\dot{\boldsymbol{r}} \times \boldsymbol{B})_{j} .
$$

Hence, the acceleration is null when the velocity is parallel to the field. Moreover, it is straightforward to obtain

$$
\sum_{j=1}^{3} B_{j} m_{j} \ddot{x}_{j}=0
$$

i.e., $\boldsymbol{N} \cdot \ddot{\boldsymbol{r}}=0$, with $N_{j}=B_{j} m_{j}$. This means that an accelerated motion can only occur provided the acceleration is perpendicular to $N$.

In order to solve Eq. (1) we look for solutions of the form $\dot{\boldsymbol{r}}=\boldsymbol{c} \exp (i \omega t)$. This leads to the following eigenvalue problem:

$$
\mathbb{A} \boldsymbol{c}=\omega \boldsymbol{c},
$$

where

$$
\mathbb{A}=i e\left(\begin{array}{ccc}
0 & B_{3} / m_{1} & -B_{2} / m_{1} \\
-B_{3} / m_{2} & 0 & B_{1} / m_{2} \\
B_{2} / m_{3} & -B_{1} / m_{3} & 0
\end{array}\right) \text {. }
$$

The eigenvalues are $\omega_{1,2}= \pm \omega_{c}$ and $\omega_{3}=0$, where $[10,11]$

$$
\omega_{c}=e \sqrt{\frac{B_{1}^{2}}{m_{2} m_{3}}+\frac{B_{2}^{2}}{m_{3} m_{1}}+\frac{B_{3}^{2}}{m_{1} m_{2}}}
$$

is the cyclotron frequency. Correspondingly, the cyclotron mass is given by $[10,12]$

$$
m_{c}=\frac{e B}{\omega_{c}}=\left(\frac{u_{1}^{2}}{m_{2} m_{3}}+\frac{u_{2}^{2}}{m_{3} m_{1}}+\frac{u_{3}^{2}}{m_{1} m_{2}}\right)^{-1 / 2}
$$

where the components of $\boldsymbol{u}=\boldsymbol{B} / B$ are the direction cosines of the magnetic field.

The first two eigenvectors give rise to an elliptical motion in a plane perpendicular to $N$. The period of such a closed orbit is $T_{c}=2 \pi / \omega_{c}$, which is used as a unit of time in the following sections. The third eigenvalue corresponds to a uniform motion along the magnetic-field direction, i.e., parallel to the first eigenvector, $\boldsymbol{c}_{1}=\boldsymbol{u}$. In principle, the electron moves along a helix which is a superposition of the elliptical and the linear motions. If the initial conditions lead to the excitation of the two motions, then the electron moves uniformly along $N$, and the motion along $\boldsymbol{B}$ is a superposition of a uniform and an oscillatory motion. Figure 1 shows the main differences between the isotropic and anisotropic cases. In the first case, $\boldsymbol{N} \| \boldsymbol{B}$ and the ellipse is a circle on a plane perpendicular to $\boldsymbol{B}$. In the second case, it is apparent that the ellipse is on a plane not perpendicular to the magnetic-field direction [13].

\section{B. The anisotropic diamagnetic Kepler problem}

When an electron with anisotropic mass, as described in the previous section, interacts with a single positive charge in the presence of a magnetic field $\boldsymbol{B}=B \boldsymbol{u}$, the equations of motion
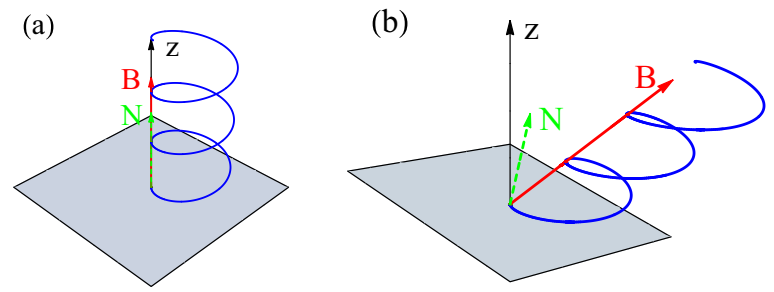

FIG. 1. (Color online) The helical trajectories of an electron with (a) isotropic and (b) anisotropic mass subject to a magnetic field. The thick solid (dashed) arrow represents the magnetic field (the vector $N$ perpendicular to the shaded plane).

read

$$
m_{j} \ddot{x}_{j}=-\frac{e^{2} x_{j}}{4 \pi \varepsilon r^{3}}-e B(\dot{\boldsymbol{r}} \times \boldsymbol{u})_{j},
$$

with $\varepsilon$ being the absolute value of the static dielectric permittivity of the medium through which the electron moves. This parameter is taken to be homogeneous and isotropic. By introducing new variables $\tilde{\boldsymbol{r}}=\boldsymbol{r} / \lambda, \tilde{t}=t / T_{c}, \mu_{j}=m_{j} / m_{c}$, with

$$
\lambda=\sqrt[3]{\frac{\pi m_{c}}{\varepsilon B^{2}}},
$$

the equations of motion become

$$
\mu_{j} \frac{d^{2} \tilde{x}_{j}}{d \tilde{t}^{2}}=-\frac{\tilde{x}_{j}}{\tilde{r}^{3}}-2 \pi\left(\frac{d \tilde{\boldsymbol{r}}}{d \tilde{t}} \times \boldsymbol{u}\right)_{j} .
$$

\section{The energy scale}

The mechanical energy of the electron is given by

$$
E=\sum_{j=1}^{3} \frac{m_{j} \dot{x}_{j}^{2}}{2}-\frac{e^{2}}{4 \pi \varepsilon r} .
$$

Hence, the dimensionless energy $\mathcal{E}_{c}=E / E_{0}$, with $E_{0}=$ $e^{2} /(4 \pi \varepsilon \lambda)$, is given by

$$
\mathcal{E}_{c}=\sum_{j=1}^{3} \frac{\mu_{j}}{2}\left(\frac{d \tilde{x}_{j}}{d \tilde{t}}\right)^{2}-\frac{1}{\tilde{r}} .
$$

Here we note that in order to compare with quantummechanical approaches, the energy is better measured in units of the transversal effective Rydberg $R y^{*}=e^{4} m_{\perp} /\left(32 \pi^{2} \varepsilon^{2} \hbar^{2}\right)$, and is given by $\mathcal{E}_{q}=E / R y^{*}$. Therefore, by introducing the dimensionless measure of the magnetic-field strength $\gamma=$ $\hbar e B /\left(2 R y^{*} m_{\perp}\right)$, we obtain

$$
\mathcal{E}_{c}=\mathcal{E}_{q} \gamma^{-2 / 3}\left(\frac{\pi^{2} m_{c}}{2 m_{\perp}}\right)^{1 / 3}
$$

\section{Equations for silicon}

Silicon has the diamond crystal structure and the Cartesian coordinates are naturally chosen along the sides of a cubic crystallographic cell. The conduction band has six equivalent valleys along the $\Gamma-X$ lines, and the effective mass is diagonal in the Cartesian system of coordinates. In each valley, the effective mass along the $\Gamma-X$ line is called the longitudinal 
mass and its value is denoted $m_{\|}=0.9163 m_{0}$, where $m_{0}$ is the bare electron mass. Moreover, the effective masses along the transversal directions are identical and their common value is given by $m_{\perp}=0.1905 m_{0}$. Hence, the anisotropy is given by the ratio $\alpha=m_{\perp} / m_{\|}=0.2079$. The relative dielectric permittivity is taken to be $\varepsilon / \varepsilon_{0}=11.4$.

In this work the energy of the donor states is referred to the conduction-band minimum and the intervalley coupling is disregarded. Thus the role of each valley depends on the angle $\theta_{B}$ between the magnetic field and the longitudinal direction of the valley. Therefore, to simplify the calculations, one may choose the $z$-axis direction along the longitudinal direction of the valley under investigation, with the $x-y$ plane containing the transversal directions. According to Sec. II A, $m_{1}=m_{2}=$ $m_{\perp}$ and $m_{3}=m_{\|}$, and the cyclotron mass is given by

$$
m_{c}=\frac{m_{\perp}}{\sqrt{\alpha \sin ^{2}\left(\theta_{B}\right)+\cos ^{2}\left(\theta_{B}\right)}} .
$$

If the magnetic field is applied along the [100] direction of silicon, then the magnetic field is parallel (perpendicular) to the longitudinal direction of two (four) valleys. Taking $\theta_{B}=0^{\circ}$ and $\theta_{B}=90^{\circ}$, the cyclotron mass is given by $m_{c}=m_{\perp} \approx$ $0.1905 m_{0}$ and $m_{c}=\sqrt{m_{\perp} m_{\|}} \approx 0.4178 m_{0}$, respectively. In contrast, when the field is along the [111] direction of silicon, the six valleys are equivalent and $\theta_{B}=\arccos (1 / \sqrt{3}) \approx$ $54.7^{\circ}$. Then the cyclotron mass is $m_{c}=m_{\perp} \sqrt{3 /(2 \alpha+1)} \approx$ $0.2773 m_{0}$.

\section{E. Treatment of the Coulomb singularity}

The numerical solution of Eq. (9) involves two main difficulties. On the one hand, the Coulomb force diverges as the inverse square of the distance to the origin. On the other hand, according to Eq. (11), the electron speed diverges as the inverse square root of the distance to the origin. This implies that the magnetic force is very intense when the electron is nearby the impurity. These difficulties are of particular concern in the present work, because the calculations focus on close orbits described by the electron after departure from the vicinity of the impurity center. When the effective mass is isotropic, one may overcome the issues by introducing a rotating reference frame, semiparabolic coordinates, and a position-dependent time scaling [8]. In the anisotropic case, as described below, the equations may be improved through a similar time-scaling transformation.

To regularize Eq. (11), one may introduce a scaled time $\tau$, such that

$$
\frac{d \tilde{t}}{d \tau}=\tilde{r}
$$

with $\tilde{t}(0)=0$, i.e.,

$$
\tilde{t}=\int_{0}^{\tau} \tilde{r}\left(\tau^{\prime}\right) d \tau^{\prime}
$$

The transformed equation reads

$$
\sum_{j=1}^{3} \frac{\mu_{j}}{2}\left(\frac{d \tilde{x}_{j}}{d \tau}\right)^{2}=1+\mathcal{E}_{c} \tilde{r}
$$

and shows that the new speed remains finite near the origin. In turn, Eq. (9) becomes

$$
\mu_{j}\left(\frac{d^{2} \tilde{x}_{j}}{d \tau^{2}}-\frac{1}{2 \tilde{r}} \frac{d \tilde{r}}{d \tau} \frac{d \tilde{x}_{j}}{d \tau}\right)=-\frac{\tilde{x}_{j}}{\tilde{r}^{2}}-2 \pi \sqrt{\tilde{r}}\left(\frac{d \tilde{\boldsymbol{r}}}{d \tau} \times \boldsymbol{u}\right)_{j} .
$$

Here the term associated with the Coulomb force presents a weaker singularity and the term due to the magnetic field is negligible near the origin.

Regarding the initial conditions at $\tau=0$, the singularity prevents us from considering a departure of the electron from the origin. Therefore, a small but finite value of the initial distance to the origin $\tilde{r}(0)=\tilde{r}_{0}$ should be used. The initial position is then given by $\tilde{\boldsymbol{r}}(0)=\tilde{r}_{0}\left(\sin \left(\theta_{r}\right) \cos \left(\phi_{r}\right), \sin \left(\theta_{r}\right) \sin \left(\phi_{r}\right), \cos \left(\theta_{r}\right)\right)$, where $\theta_{r}$ and $\phi_{r}$ are the polar and azimuthal angles. In analogy, the initial velocity is taken as $\frac{d \tilde{r}}{d \tau}(0)=$ $\tilde{v}_{0}\left(\sin \left(\theta_{v}\right) \cos \left(\phi_{v}\right), \sin \left(\theta_{v}\right) \sin \left(\phi_{v}\right), \cos \left(\theta_{v}\right)\right)$, where, according to Eq. (16), the initial speed is given by

$$
\tilde{v}_{0}=\sqrt{\frac{2\left(1+\mathcal{E}_{c} \tilde{r}_{0}\right)}{\mu_{\perp} \sin ^{2}\left(\theta_{v}\right)+\mu_{\|} \cos ^{2}\left(\theta_{v}\right)}} .
$$

In the isotropic case, one may choose $\phi_{r}=\phi_{v}$ and $\theta_{r}=\theta_{v}$. This is because the dominant force near the origin is a central field. In silicon, the anisotropy presents axial symmetry and the first condition still holds. However, the polar angle undergoes fast changes near the origin. This means that an electron that is radially launched from the surface $\tilde{r}=\tilde{r}_{0}$ does not necessarily come from the origin (if negative values of $\tau$ were considered). Therefore, for each value of $\theta_{v}$, one must consider the special values of $\theta_{r}$ that correspond to trajectories coming (essentially) from the impurity center.

\section{NUMERICAL RESULTS}

To investigate the closed orbits of the electron, Eq. (17) is solved by launching the electron from a distance $\tilde{r}_{0}=10^{-6}$. We first deal with the isotropic case. Then, the effects of anisotropy for different magnetic-field directions and electron energies are discussed, including a comparison with experimental data.

\section{A. Isotropic reference case}

The case of an electron with isotropic effective mass corresponds to $\alpha=1$. Without losing generality, one may choose the magnetic field along the $z$-axis direction. The shape and duration of the orbits is independent of the initial azimuthal angle $\phi_{v}$, but different results are expected as the initial polar angle $\theta_{v}$ is varied. In view of the symmetry, the calculations have been performed for $0 \leqslant \theta_{v} \leqslant 90^{\circ}$, with a uniform step of $1^{\prime \prime}$. In this way, a set of orbits where the electron returns to the near vicinity of the impurity center is obtained. For short, such trajectories are called closed orbits.

When seen from a reference frame rotating around the $z$ axis at the Larmor frequency [8], $\omega_{c} / 2$, the eight most important orbits for $\mathcal{E}_{c}=0$ have the shapes displayed in Fig. 2. The horizontal and vertical axes correspond to the cylindrical coordinates $\tilde{\rho}=\tilde{x} \cos (\pi \tilde{t})+\tilde{y} \sin (\pi \tilde{t})$ and $\tilde{z}$. We note that 


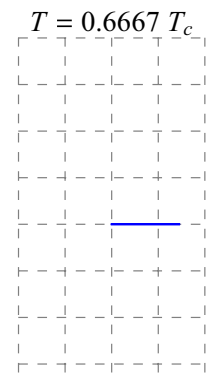

(a)
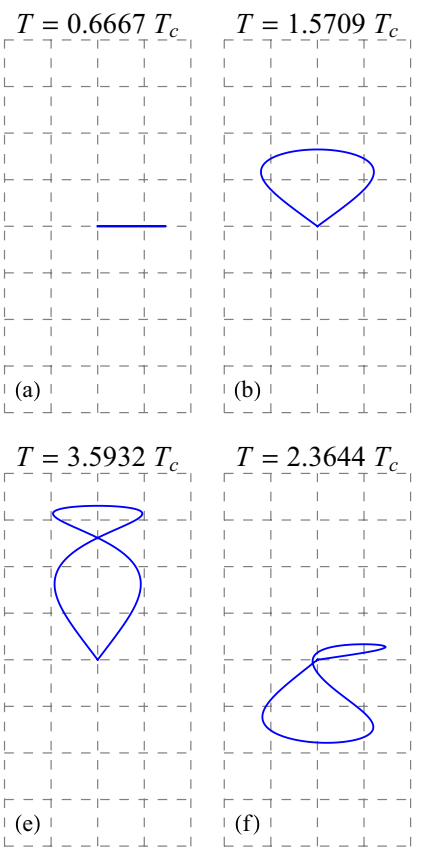

(b)
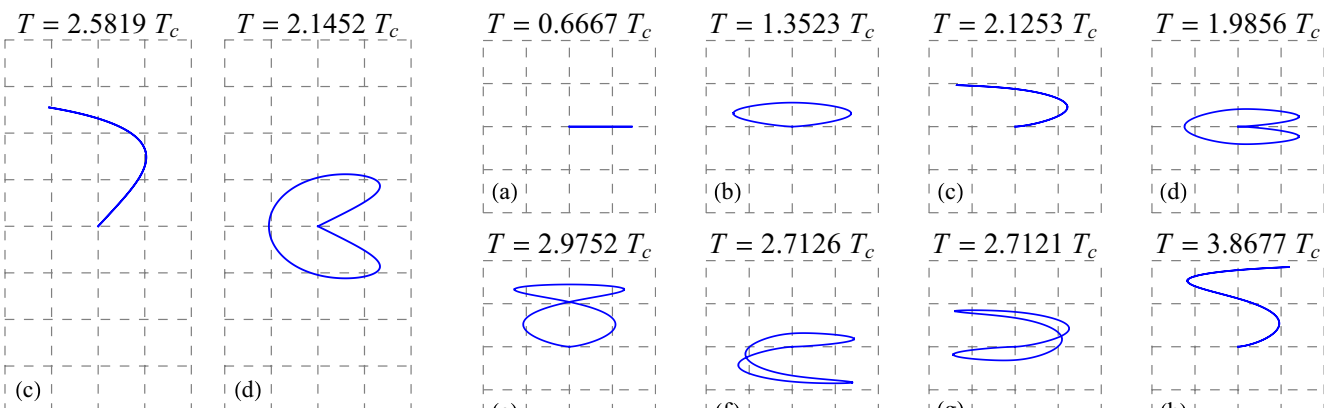

$T=2.9752 T_{c}$
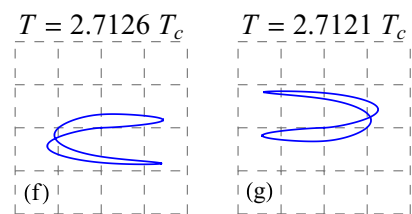

(d)

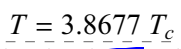

(d)
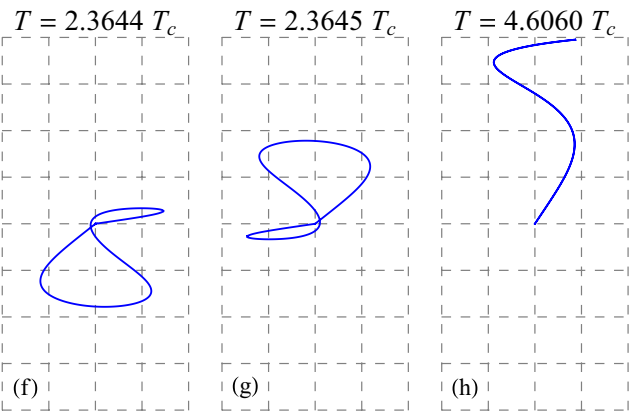

FIG. 2. (Color online) The eight most important closed orbits with $\mathcal{E}_{c}=0$ in the isotropic case. Each curve is labeled by the flight time in units of the cyclotron period. The origin is at the center of the graph and the horizontal and vertical axes correspond to the cylindrical coordinates $\tilde{\rho}$ and $\tilde{z}$, respectively. The magnetic field is along the $\tilde{z}$-axis direction and the spacing between grid lines is 0.4 .

all shapes and flight times are in good agreement with the results reported by $\mathrm{Du}$ and Delos [7], and the corresponding periods agree with those experimentally found by Main et al. [5] in the optical spectra of hydrogen. Also, comparing with Figs. 3, 4, and 5 of Ref. [8], Figs. 2(a), 2(b), 2(c), 2(e), and 2(h) correspond to the orbits from $\mathrm{I}_{1}$ to $\mathrm{I}_{5}$, while Figs. 2(d) and 2(f) are for the orbits $\mathrm{IIb}_{0}$ and $\mathrm{IIa}_{1}$, respectively. In particular, Fig. 2(a) is produced when the electron is launched at $\theta_{v}=90^{\circ}$. In such a case, the electron describes a plane trajectory and visits the origin at integer multiples of the Garton-Tomkins period [5,14], namely $\frac{2}{3} T_{c}$.

\section{B. Anisotropic case}

To begin the analysis of the effects of anisotropy in silicon, we consider the case where the magnetic field is parallel to the longitudinal direction of the valley. In this case, because of the axial symmetry, the shape and duration of the orbits do not depend on the launching azimuthal angle $\phi_{v}$, and one may limit the analysis to those values of the launching polar angle $\theta_{v}$ between $0^{\circ}$ and $90^{\circ}$.

The numerical results are obtained for $\mathcal{E}_{c}=0$, by varying $\theta_{v}$ with step $0.1^{\prime \prime}$. Closed orbits lasting less than five cyclotron periods only occur for launching polar angles very close to $90^{\circ}$. This means that the electron will not be able to come back within five cyclotron periods unless it departs along a direction with nearly minimum inertia.

The calculated orbits of donor electrons with energy $\mathcal{E}_{c}=0$, in silicon subject to a magnetic field along the longitudinal direction of the valley, are displayed in Fig. 3. In this case, the orbits may be analyzed from a reference frame rotating (g)

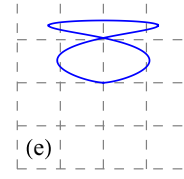

(f)

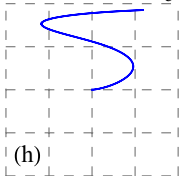

FIG. 3. (Color online) As for Fig. 2, in silicon, with the magnetic field along the longitudinal direction of the valley. Only the eight orbits resembling those in Fig. 2 are shown.

at the Larmor frequency, and only those trajectories resembling the curves in Fig. 2 are shown. One may note two main effects of the anisotropy. On the one hand, except for the GartonTomkins period, the anisotropy changes the duration of the closed orbits. On the other hand, since the spacing between grid lines is 0.4 in both figures, it is apparent that the anisotropy shrinks the orbits along the longitudinal direction of the valley.

The effects of the anisotropy in silicon should be stronger when the magnetic field is not parallel to the longitudinal direction of the valley. In such cases, the rotating reference frame is of little use and one has to deal with three-dimensional orbits. To be concrete, we deal with the configuration where the magnetic field is applied along the [111] direction. Among the possibilities, this case is quite simple because the six valleys of the conduction band are equivalent (see Sec. II D). Numerical results were obtained for $\mathcal{E}_{c}=0$ and $\phi_{v}=45^{\circ}$, by varying $\theta_{v}$ with step $1^{\prime \prime}$ in the range between $0^{\circ}$ and $180^{\circ}$. In this way, the electron is launched in the plane containing the $z$ axis and the field direction. The three main closed orbits are shown in Fig. 4. The orbit displayed in Fig. 4(a) resembles the one in Fig. 2(a), and has a duration near the Garton-Tomkins period. However, the plane perpendicular to $N$ is not horizontal and the orbit is not exactly in the plane. In Figs. 4(b) and 4(c) one may note that trajectories are elongated in the direction of the magnetic field. When the electron is far from the impurity, the field affects the trajectory as to describe almost elliptical arcs whose plane is perpendicular to the vector $\boldsymbol{N}$. This is essentially the behavior displayed by the helical trajectory in Fig. 1(b).

\section{Comparison with experiment}

Up to now we have seen that anisotropy affects the classical orbits of a conduction electron in silicon subject to a
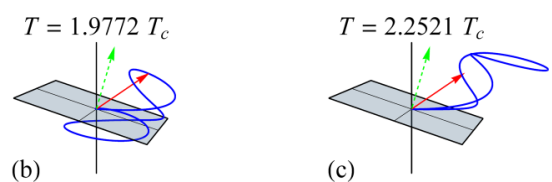

FIG. 4. (Color online) Closed orbits with $\mathcal{E}_{c}=0$, in silicon subject to a magnetic field along the [111] direction. Only the first three periods obtained for $\phi_{v}=45^{\circ}$ are shown. The vertical line is the $z$ axis and the solid (dashed) arrow represents the magnetic field (the vector $N$ in Sec. II A, perpendicular to the shaded plane). The side of the shaded square is 0.8 units long.

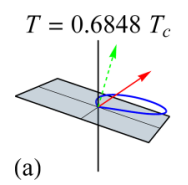


magnetic field, and the effects depend on the angle between the magnetic-field direction and the longitudinal direction of the band valley. A comparison with experimental data is naturally in order. Thus we consider a magnetic field of strength $B=4 \mathrm{~T}$ applied along the [111] direction, corresponding to the results reported in Fig. 3(a) of Ref. [9]. The cyclotron period is $T_{c} \approx 2.4766 \mathrm{ps}$, and the oscillating part of the spectrum extends over a range of width $100 \mathrm{~cm}^{-1}$ above the ionization threshold. This corresponds to dimensionless values of the electron energy satisfying $0 \leqslant \mathcal{E}_{c} \leqslant 8.8$.

Under these conditions, the periods and the shapes of the orbits are expected to change noticeably as the energy varies within such a range. Consequently, the standard Fourier transform is not the best tool to obtain the periods of the main semiclassical orbits. In fact, the sinusoidal components of the absorption strength depend on the antiderivative of the orbit period (see Eq. (2) in Ref. [15]). When the periods are essentially constant over the considered spectrum, the oscillations are harmonic and the Fourier transformation works well $[5,16,17]$. Unfortunately, this is not the experimental case and the reported periods should be considered as rough estimates: $T=2.15,5.05,6.88,8.30,11.54 \mathrm{ps}$. From the theoretical point of view, such periods might be found in some agreement with the periods calculated for $\mathcal{E}_{c} \approx 4$. According to Eq. (12), this corresponds to $\mathcal{E}_{q} \approx 58.6 \mathrm{~cm}^{-1}$, a value which is near the midpoint of the spectrum of Ref. [9].

The calculations for $\mathcal{E}_{c}=4$ are displayed in Fig. 5. The launching angles $\phi_{v}$ and $\theta_{v}$ vary between $0^{\circ}$ and $180^{\circ}$ with steps $5^{\circ}$ and $0.5^{\prime \prime}$, respectively. The dots are for orbits where the electron comes back to the origin, reentering a sphere of radius 0.01 , centered at the origin. The wide lines correspond to the experimental periods $T / T_{c} \approx 0.87,2.04,2.78,3.35,4.66$, and the theoretical results are found to be in reasonable agreement with the experiment. In fact, a series of closed-orbit periods

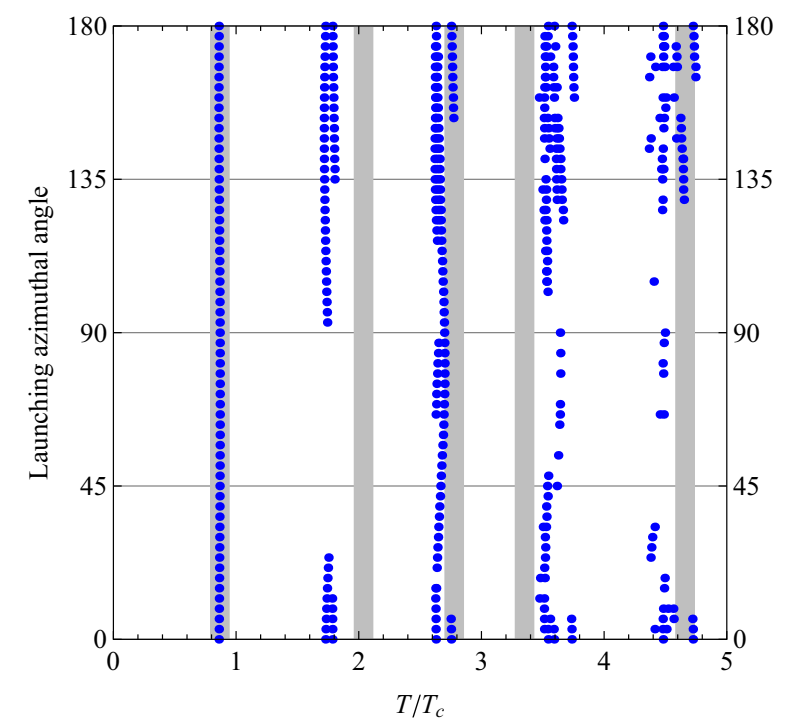

FIG. 5. (Color online) The periods of the closed orbits with $\mathcal{E}_{c}=$ 4 , as a function of the azimuthal angle $\phi_{v}$, in silicon, with the magnetic field along the [111] direction. The dots correspond to the solutions of Eq. (9) with $\mu_{1}=\mu_{2}=0.6870$ and $\mu_{3}=3.3043$. Only orbits where the electron reenters a sphere of radius 0.01 , centered at the origin, are included. The thick lines are for the experimental data in Ref. [9].

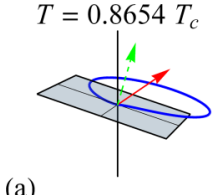

(a)

FIG. 6. (Color online) As for Fig. 4, with $\mathcal{E}_{c}=4$ and $\phi_{v}=45^{\circ}$.

that partially reproduce the peak positions in the Fourier transform of the experimental spectrum has been found. The agreement is expected to improve if the contribution of different energy values is taken into account, at a much larger computational effort. Quantum-mechanical calculations should also contribute to a deeper understanding of the spectrum. Of course, new experimental measurements and data processing would be most valuable.

The shapes of some closed orbits corresponding to the points of Fig. 5 are shown in Fig. 6, for the case of an electron launched at an azimuthal angle of $45^{\circ}$. After comparing with Fig. 4, it is apparent that the periods and shapes depend on the energy. By changing the value of $\phi_{v}$ to $135^{\circ}$, the orbits take the shapes displayed in Fig. 7. It may be seen that the results depend on the launching azimuthal angle as well. We remark that the optical spectrum arises from the combination of the closed electron orbits associated with different energies and launching directions. Moreover, the contribution of each polar angle $\theta_{v}$ should be weighted by a factor $\sin \left(\theta_{v}\right)$, in order to compensate the higher density of $\phi_{v}$ values. Nevertheless, Figs. 6 and 7 illustrate the electron dynamics related with a semiclassical interpretation of the experimental results.

Regarding the experimental conditions required for the manifestation of the classical orbits in the optical spectrum, we note that the radius of the main closed orbits investigated in this work is of the order of the length unit $\lambda$, which is given by Eq. (8). The dependence of $\lambda$ on the magnetic-field strength in silicon, with the field applied along the [111] direction, is displayed in Fig. 8(a). First we note that orbits of radius larger than $1 \mu \mathrm{m}$ occur for magnetic fields between 0.001 and $0.1 \mathrm{~T}$, while $\lambda \approx 79 \mathrm{~nm}$ when $B=4 \mathrm{~T}$. This poses the problem of whether the impurities in the silicon sample may be treated as if they were isolated.

If the mean impurity spacing is larger than $20 \lambda$, then the impurities may be regarded as isolated. This threshold is shown by the thick line in Fig. 8(b), as a function of the magneticfield strength. The dark region below the line corresponds to the regime where the classical orbits manifest themselves. When $B=4 \mathrm{~T}$, the donor concentration should be less than $3 \times 10^{11} \mathrm{~cm}^{-3}$, in agreement with the experimental conditions in the work of Chen et al. [9]. It is also apparent that, for magnetic fields weaker than $0.1 \mathrm{~T}$, the concentration should be less than $1 \times 10^{8} \mathrm{~cm}^{-3}$. Such a low doping concentration may

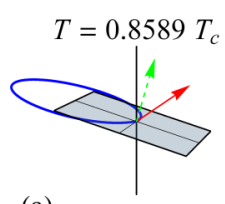

(a)

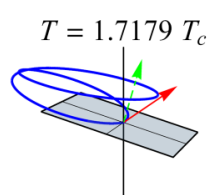

(b)

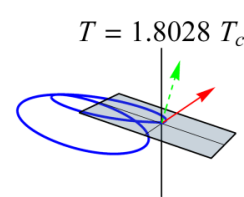

(c)
FIG. 7. (Color online) As for Fig. 6, with $\mathcal{E}_{c}=4$ and $\phi_{v}=135^{\circ}$. 

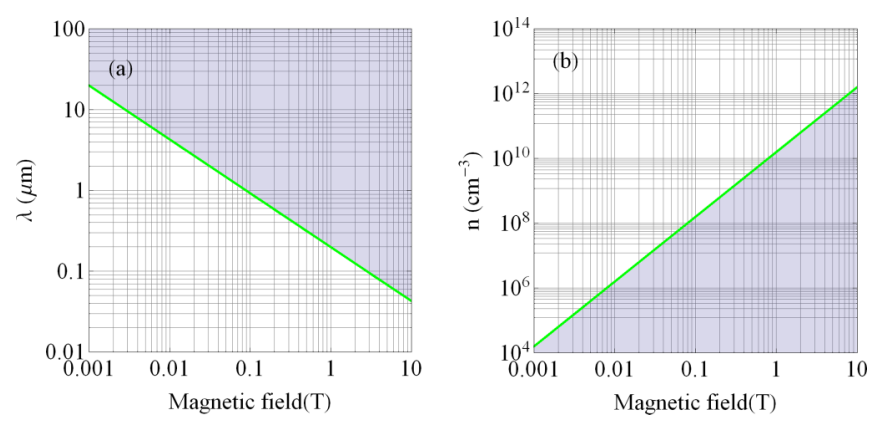

FIG. 8. (Color online) (a) The radius of the main classical orbits as a function of the magnetic-field strength. (b) Mean donor concentration that corresponds to a cubic array of donor impurities with lattice constant $20 \lambda$.

be difficult to detect by optical measurements. This leads to a lower bound for the applied magnetic field that depends on the sensitivity of the spectroscopic system. At the same time, for the validity of the semiclassical approach, the unit length $\lambda$ should be greater than 10 times the effective Bohr radius of the donor impurity. Then, the magnetic-field intensity should not be greater than $16 \mathrm{~T}$.

\section{CONCLUSIONS}

The periods and the shapes of closed orbits of an electron with anisotropic mass interacting with a Coulomb center in the presence of a magnetic field have been shown to depend on the energy and the magnetic-field direction. After a qualitative analysis of the periods and shapes of the orbits at the ionization threshold, we have chosen the classical electron energy near the middle of the energy range where the oscillations of the experimental absorption occur. We have found reasonable agreement with available experimental data. However, a careful analysis has revealed that the Fourier transform of the optical spectra associated with donors in a semiconductor subject to a magnetic field of $4 \mathrm{~T}$ may not give the actual periods of relevant closed orbits. This is because the periods change over the energy range of the investigated spectra [15].

We hope this work will motivate further experimental studies. To facilitate this, requirements for the donor-doping concentration and the magnetic-field strength have been given. In the meantime, a deeper investigation of the classical orbits in the anisotropic diamagnetic Kepler problem and quantum mechanical calculations [18-21] of the optical spectrum are in progress.

\section{ACKNOWLEDGMENTS}

A.B.-A. and G.V.B.d.S. are thankful to the University of Wollongong for their warm hospitality and financial support (under 2012/13 UIC International Links Scheme). We are also grateful to the Brazilian Agencies FAPESP and CNPq (including a student fellowship under Science Without Borders), as well as to the Graduate Program POSMAT/Unesp, for financial support. The work was supported by the Australian Research Council. R.A.L. acknowledges useful discussions with S.-C. Shen.
[1] W. R. S. Garton and F. S. Tomkins, Astrophys. J. 158, 839 (1969).

[2] D. Wintgen, J. Phys. B 20, L511 (1987).

[3] D. Delande, Phys. Scr. 34, 52 (1991).

[4] J. Main and G. Wunner, J. Phys. B 27, 2835 (1994).

[5] J. Main, G. Wiebusch, A. Holle, and K. H. Welge, Phys. Rev. Lett. 57, 2789 (1986).

[6] A. Holle, J. Main, G. Wiebusch, H. Rottke, and K. H. Welge, Phys. Rev. Lett. 61, 161 (1988).

[7] M. L. Du and J. B. Delos, Phys. Rev. A 38, 1896 (1988).

[8] D. Wintgen and H. Friedrich, Phys. Rev. A 36, 131 (1987).

[9] Z. Chen, W. Zhou, B. Zhang, C. H. Yu, J. Zhu, W. Lu, and S. C. Shen, Phys. Rev. Lett. 102, 244103 (2009).

[10] J. Sólyom, Fundamentals of the Physics of Solids, Vol. 2 (Springer, Berlin, 2009).

[11] M. P. Marder, Condensed Matter Physics, 2nd ed. (John Wiley \& Sons, Hoboken, 2010).

[12] W. Shockley, Phys. Rev. 90, 491 (1953).
[13] G. E. Smith, L. C. Hebel, and S. J. Buchsbaum, Phys. Rev. 129, 154 (1963).

[14] J. A. C. Gallas and R. F. O’Connell, J. Phys. B 15, L75 (1982).

[15] M. L. Du and J. B. Delos, Phys. Rev. Lett. 58, 1731 (1987).

[16] W. Hogervorst, A. Kips, K. Karremans, T. van der Veldt, G. Kuik, and W. Vassen, in Atoms and Molecules in Strong External Fields, edited by P. Schmelcher and W. Schweizer (Kluwer Academic, New York, 2002), pp. 109-119.

[17] J. Main, G. Wunner, V. A. Mandelshtam, and H. S. Taylor, in Atoms and Molecules in Strong External Fields, edited by P. Schmelcher and W. Schweizer (Kluwer Academic, New York, 2002), pp. 215-221.

[18] R. A. Faulkner, Phys. Rev. 184, 713 (1969).

[19] Y.-M. Mu, J.-P. Peng, P.-L. Liu, S.-C. Shen, and J.-B. Zhu, Phys. Rev. B 48, 10864 (1993).

[20] A. Bruno-Alfonso, R. A. Lewis, G. Q. Hai, and R. E. M. Vickers, J. Low Temp. Phys. 159, 226 (2010).

[21] R. A. Lewis, A. Bruno-Alfonso, G. V. B. de Souza, R. E. M. Vickers, J. A. Colla, and E. Constable, Sci. Rep. 3, 3488 (2013). 